\title{
Adaptive system of intelligent environment based on neural networks
}

\author{
Pavel L. Nikolaev \\ Moscow Aviation Institute (National Research University) \\ Moscow, Russia \\ npave189@gmail.com
}

\begin{abstract}
This paper describes the concept of adaptive system of intelligent environment for smart homes and intelligent buildings. This intelligent system is based on one of the methods of artificial intelligence and machine learning - artificial neural networks. The key feature of the proposed system is the recognition of current human activity and the switching of the intelligent environment system in the most optimal mode for performing this activity. These operations are performed by neural networks.
\end{abstract}

Keywords-intelligent environment; smart home; intelligent building; artificial intelligence; machine learning, artificial neural networks; deep learning; convolutional neural networks.

\section{INTRODUCTION}

A distinctive feature of smart homes (intelligent buildings) is the availability of a unified automated control and monitoring system for all components (lighting system, microclimate system, security system and others) of an apartment, house or building [1]. The main objectives of the application of these technologies are improvement living and working conditions, safety of inhabitants and increase energy saving. Energy saving is an important task because according to global statistics $30 \%$ of total energy consumption and $60 \%$ of electricity consumption are spent on buildings [2].

In the case where the system consists of a large number of components and operates with a large amount of data it is very difficult, long and sometimes costly to create effective work algorithms. In addition to integrators, sometimes users also have to deal with such tasks for customizing intelligent management systems on their own. In addition, such systems can't be called truly smart because they perform deterministic scenarios and can't independently make decisions in atypical situations. Therefore, the real smart home systems should have the likeness of an artificial intelligence (AI) that can independently make decisions and regulate the activities of all systems of a building or a house depending on the constantly changing conditions and needs of users. Methods of AI and machine learning (ML) can be effectively used for such tasks as building energy management and energy efficiency [3], improving the security of both systems and users, making systems adaptable to users (it will be most useful for people with disabilities).
We will use the concept of "intelligent environment" for smart homes and intelligent buildings which are based on methods of AI and ML. At the same time, such an intelligent environment can surround users both at home and in the workplace.

In this study we consider the adaptive system of intelligent environment (ASIE) which is based on an artificial neural network (ANN). ANN is one of the methods of artificial intelligence and machine learning. This method has recently received a new development and finds increasing application in various fields, including in the field of building automation.

Basically, the work in the field of application of ANN in systems of smart homes or intelligent buildings is centered around predicting the energy consumption of buildings [3-5] and the management of various house systems by predicting user actions based on accumulated data on their previous activities (for example, switching on and off lighting devices in [6]).

The main function and main distinctive feature of the neural network intelligent system proposed in this article is the ability to recognize the current activity of the user. Next, the system must adapt to this activity, identifying and using the most optimal operating modes of the composite subsystems of the intelligent environment.

\section{ARCHITECTURE OF THE ADAPTIVE SYSTEM OF INTELLIGENT ENVIRONMENT}

The scheme of organization of the proposed system of intelligent environment is depicted in Fig. 1. Let us consider its components in more detail.

The server is the main component in a typical system of smart home or intelligent building. It is equipped with special software which is responsible for the operation of the entire intelligent system. Also, the server is an intermediary between control and monitoring devices (computers, mobile devices) and controllers. User commands from control and monitoring devices to controllers and sensor data in the reverse order are transmitted via the server. It is on such servers the software of the adaptive intelligent system must be deployed. This makes it possible to integrate the proposed intelligent system over an existing control and monitoring system. Thus, it is possible to 


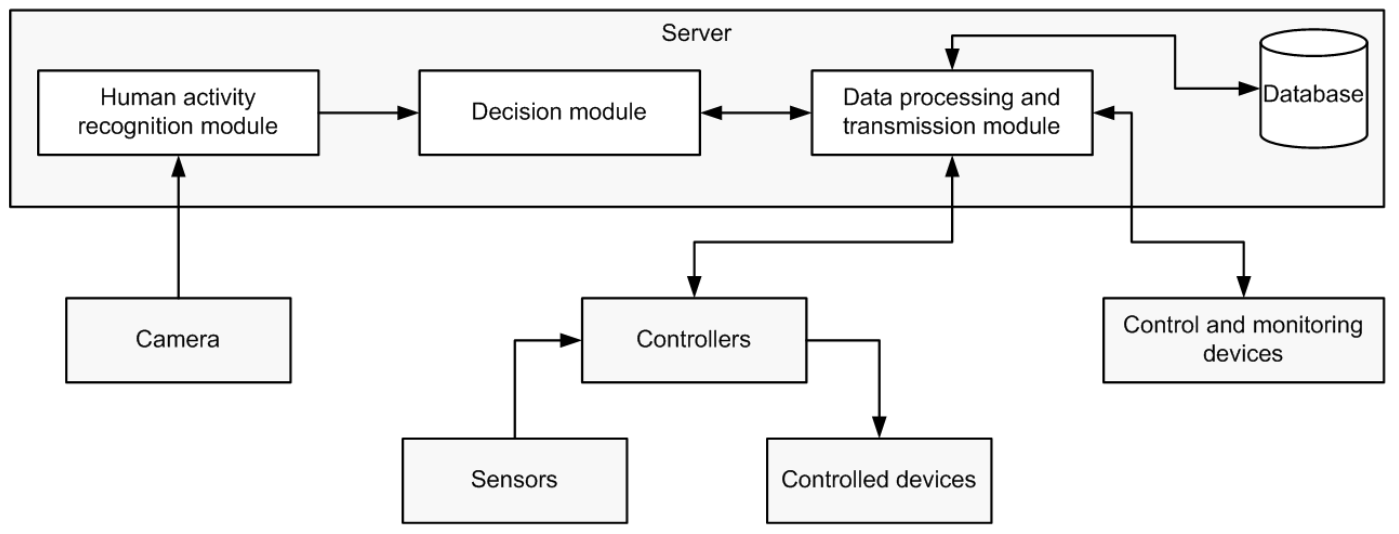

Fig. 1. Structural diagram of the intelligent environment system

introduce the adaptive system of intelligent environment in any automation project, regardless of the equipment and software used there.

The server software with the integrated intelligent environment system will consist of the following main modules:

- Database. It contains information about the state of elements of the intelligent environment for a certain period of time.

- Data processing and transmission module. This module is responsible for receiving data about the status of sensors and controlled devices and storing them in the database. It also processes user commands.

- Human activity recognition module (HAR module). Its task is to recognize the current activity of the user (reading a book, writing, working at a computer, lack of any activity, etc.) by analyzing the image of the room received from the camera.

- Decision module. This module calculates the most suitable conditions for the current activity of the user. Further, it decides how to change the operation of controlled devices. The decision is made on the basis of data on the current activity of the user, the current time of day and data from sensors located in the area where this activity is manifested.

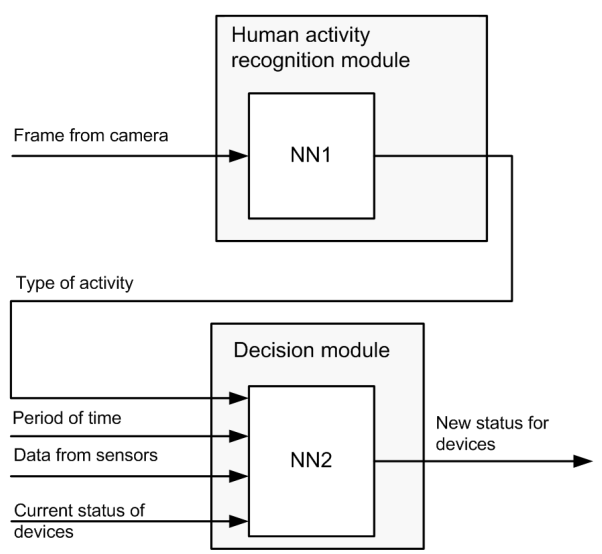

Fig. 2. Interaction diagram of neural network modules

\section{APPLICATION OF ARTIFICIAL NEURAL NETWORKS}

In the considered intelligent system we use two artificial neural networks which are the basis of HAR module and decision module (Fig. 2).

\section{A. Recognition of activities}

The human activity recognition module is the neural network (NN1) that recognizes the person's current activity on the camera image. The input of the network is a frame from the camera $(I)$ and the output of the network is the type of user activity $(A)$ :

$$
y_{1}=f(I)=A
$$

Image recognition requires deep neural network. The best solution for this task is to use a convolutional neural network (CNN). The CNN architecture was proposed by Yann LeCun [7] in 1998. However, the greatest development of convolutional neural networks has been obtained after the victory of the AlexNet network [8] in ImageNet challenge on visual recognition. Since then CNN has been effectively used in computer vision in recognizing images.

\section{B. Decision making}

The decision module is also based on the neural network (NN2). The task of this network is to define a new mode of operation for any system (for example, lighting system, temperature control system, etc.) or separate devices. The following data are input to the network:

- current user activity $(A)$;

- $\quad$ current time period $(P)$;

- data from sensors $(S)$;

- current device states at time $t(D(t))$.

The output of the network is a new mode of operation (new states of controlled devices included in this system) of any system or separate devices $(D(t+1))$ :

$$
y_{2}=f(A, S, P, D(t))=D(t+1)
$$


Further, the intelligent system must generate the necessary commands to put the devices in the desired mode.

At the first stage of development we will use a multilayer perceptron (MLP) to build the decision-making system.

\section{EXPERIMENTS}

Two neural networks have been trained - the convolutional network for image recognition and the multilayer perceptron to control lighting in the room.

Both neural networks have been implemented in Python 3.6.1. The following libraries have also been used:

- Theano 0.9.0;

- Keras 2.0.6;

- cuDNN 5.1.

We have done the calculations on the laptop with video card NVIDIA GeForce 940MX with 2 GB of video memory GDDR5. However, only $1.6 \mathrm{~GB}$ of video memory was used.

\section{A. Convolutional neural network}

During the experiments the convolutional neural network (Table 1) has been trained to recognize four types of human activity: reading, computer work, writing and playing a musical instrument (violin). The dataset consisted of 5600 images - 1400 images for each type of activity.

TABLE I. THE ARCHITECTURE OF THE CONVOLUTIONAL NEURAL NETWORK

\begin{tabular}{|c|c|c|}
\hline Layer (type) & Output Shape & Param \# \\
\hline conv2d_1 (Conv2D) & (None, 198, 198, 32) & 896 \\
\hline $\begin{array}{l}\text { activation_1 } \\
\text { (Activation) }\end{array}$ & (None, 198, 198, 32) & 0 \\
\hline $\begin{array}{l}\text { max_pooling2d_1 } \\
(\text { MaxPooling2D) }\end{array}$ & (None, 99, 99, 32) & 0 \\
\hline conv2d_2 (Conv2D) & (None, 97, 97, 32) & 9248 \\
\hline $\begin{array}{l}\text { activation_2 } \\
\text { (Activation) }\end{array}$ & (None, 97, 97, 32) & 0 \\
\hline $\begin{array}{c}\text { max_pooling2d_2 } \\
\text { (MaxPooling2D) }\end{array}$ & (None, $48,48,32$ ) & 0 \\
\hline conv2d_3 (Conv2D) & (None, 46, 46, 64) & 18496 \\
\hline $\begin{array}{l}\text { activation_3 } \\
\text { (Activation) }\end{array}$ & (None, $46,46,64)$ & 0 \\
\hline $\begin{array}{l}\text { max_pooling2d_3 } \\
\text { (MaxPooling2D) }\end{array}$ & (None, 23, 23, 64) & 0 \\
\hline conv2d_4 (Conv2D) & (None, $21,21,128$ ) & 73856 \\
\hline $\begin{array}{l}\text { activation_4 } \\
\text { (Activation) }\end{array}$ & (None, $21,21,128$ ) & 0 \\
\hline $\begin{array}{c}\text { max_pooling2d_4 } \\
\text { (MaxPooling2D) }\end{array}$ & (None, $10,10,128)$ & 0 \\
\hline flatten_1 (Flatten) & (None, 12800) & 0 \\
\hline dense_1 (Dense) & (None, 64) & 819264 \\
\hline $\begin{array}{l}\text { activation_5 } \\
\text { (Activation) }\end{array}$ & (None, 64) & 0 \\
\hline dropout_1 (Dropout) & (None, 64) & 0 \\
\hline dense_2 (Dense) & (None, 4$)$ & 260 \\
\hline $\begin{array}{l}\text { activation_66 } \\
\text { (Activation) }\end{array}$ & (None, 4$)$ & 0 \\
\hline
\end{tabular}

\begin{tabular}{|c|c|c|}
\hline Layer (type) & Output Shape & Param \# \\
\hline \multicolumn{3}{|c|}{ Total params: 922,020} \\
\hline \multicolumn{3}{|c|}{ Trainable params: 922,020} \\
\hline \multicolumn{3}{|c|}{ Non-trainable params: 0} \\
\hline
\end{tabular}

The algorithm for learning the convolutional network is Adam with default hyperparameters. The loss function is the categorical entropy.

We used ELU activataion function after the convolutional and fully connected layers, because according to [9] its application provides faster and more accurate training of deep neural networks. The activation function softmax was used on the output layer.

The network was trained for 100 epochs. Training of the convolutional network took about 11.5 hours. Figures 3 and 4 show the accuracy and loss of the network after 100 learning epochs. The best results on the test dataset have been achieved at the 82nd epoch (Table II).

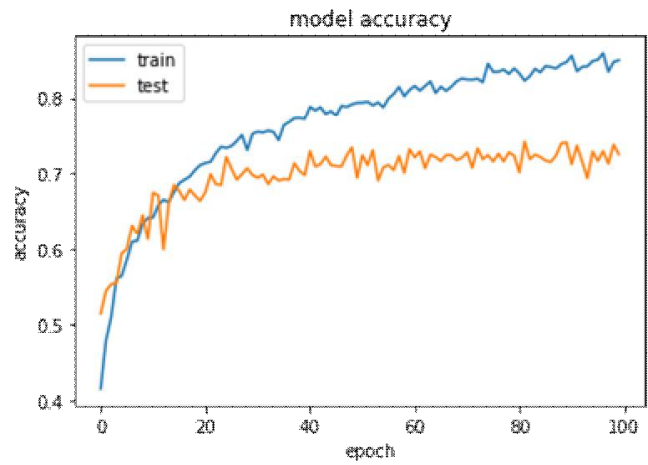

Fig. 3. Accuracy of the convolutional network

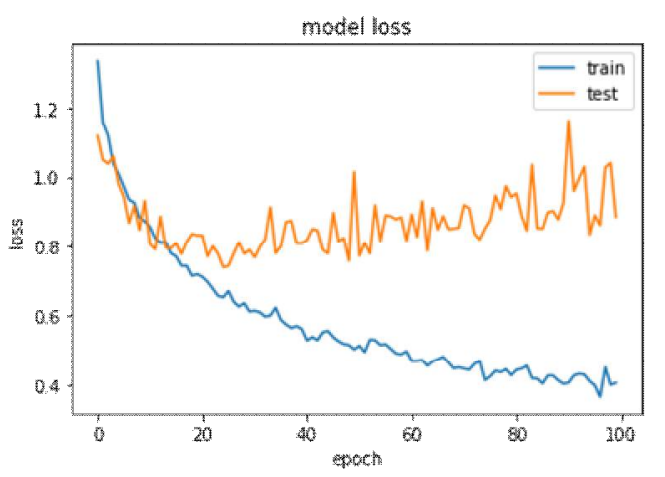

Fig. 4. Loss of the convolutional network

TABLE II. THE BEST RESULTS FOR TRAINING THE CONVOLUTIONAL NETWORK

\begin{tabular}{|c|c|c|c|}
\hline Dataset & $\begin{array}{c}\text { Number of } \\
\text { examples }\end{array}$ & Accuracy & Loss \\
\hline Training & 3920 & $82.32 \%$ & 0.4467 \\
\hline Test & 1680 & $74.23 \%$ & 0.8896 \\
\hline
\end{tabular}




\section{B. Multilayer perceptron}

The multilayer perceptron (Table 3 ) has been trained to control indoor lighting. The dataset consisted of 576 examples. The following parameters were taken into account:

- current user activity;

- time of day (day or night);

- level of illumination in the room;

- current state of four lamps.

At the output of the network was new state of these lamps.

TABLE III. THE ARCHITECTURE OF THE MULTILAYER PERCEPTRON

\begin{tabular}{|c|c|c|}
\hline Layer (type) & Output Shape & Param \# \\
\hline dense_1 (Dense) & (None, 10) & 120 \\
\hline $\begin{array}{c}\text { activation_1 } \\
\text { (Activation) }\end{array}$ & (None, 10) & 0 \\
\hline dense_2 (Dense) & (None, 4) & 44 \\
\hline $\begin{array}{c}\text { activation_2 } \\
\text { (Activation) }\end{array}$ & (None, 4) & 0 \\
\hline $\begin{array}{l}\text { Total params: 164 } \\
\text { Trainable params: } 164 \\
\text { Non-trainable params: } 0\end{array}$ & \\
\hline
\end{tabular}

The input layer of the network has consisted of 11 neurons, the hidden layer - 10 neurons and the output layer -4 neurons.

The algorithm for learning the MLP is Adam with default hyperparameters. The loss function is mean squared error.

The activation function on the hidden layer is ELU, on the output layer is sigmoid function.

The network was trained for 200 epochs. The training took about one minute. The results of the training are presented in Table 4.

TABLE IV. THE BEST RESULTS FOR TRAINING THE MULTILAYER PERCEPTRON

\begin{tabular}{|c|c|c|}
\hline Dataset & Number of examples & Loss (MSE) \\
\hline Training & 384 & 0.0031 \\
\hline Test & 192 & 0.0043 \\
\hline
\end{tabular}

\section{CONCLUSION AND FUTURE WORKS}

In this research the architecture of the adaptive system of the intelligent environment has been considered. This system can be implemented in smart homes and intelligent buildings. The principle of operation of the presented adaptive intelligent system is based on recognition of user activity and adjustment of the surrounding space to more safe, comfortable and energyefficient living and working conditions. We have decided to build the intelligent system based on artificial neural networks to perform these tasks. The convolutional network has been trained to recognize human activity. Also it has been trained the multilayer perceptron for lighting in the room.

In the future, it is planned to train the convolutional neural network to recognize more types of human activity, as well as train the second neural network to manage a large number of intelligent environment systems (microclimate control system, security system, etc.). In addition, it is necessary to achieve better accuracy of recognition by the convolutional network and to reduce the error of the multilayer perceptron.

\section{REFERENCES}

[1] P.L. Nikolaev, "The architecture of a smart home management system integrated into the cloud environment," in Programmnye produkty i sistemy (Software \& Systems), vol. 2, pp. 65-69, 2015. (In Russian)

[2] M. Manic, D. Wijayasekara, K. Amarasinghe, J.J. Rodriguez-Andina, "Building energy management systems: The age of intelligent and adaptive buildings," in IEEE Industrial Electronics Magazine, vol. 10 (1), pp. 25-39, 2016.

[3] M. Manic, K. Amarasinghe, J.J. Rodriguez-Andina, C. Rieger, "Intelligent Buildings of the Future: Cyberaware, Deep Learning Powered, and Human Interacting," in IEEE Industrial Electronics Magazine, vol. 10 (4), pp. 32-49, 2016.

[4] E. Mocanu, P.H. Nguyen, M. Gibescu, W.L. Kling, "Deep Learning for Estimating Building Energy Consumption", in Sustainable Energy, Grids and Networks, vol. 6. pp. 91-99, 2016.

[5] D. Marino, K. Amarasinghe, M. Manic, "Building energy load forecasting using deep neural networks", in Proceedings of the 42nd Annual Conference of the IEEE Industrial Electronics Society (IECON).

[6] C. Machado, J.A. Mendes. "Automatic Light Control in Domotics using Artificial Neural Networks", in International Journal of Electrical, Computer, Energetic, Electronic and Communication Engineering, vol. 2 (8), pp. 1607-1612, 2008.

[7] Y. LeCun, L. Bottou, Y. Bengio, P. Haffner, "Gradient-based learning applied to document recognition", IEEE, 1998.

[8] A. Krizhevsky, I. Sutskever, G.E. Hinton. "Imagenet classification with deep convolutional neural networks", in Advances in neural information processing systems, pp. 1097-1105, 2012.

[9] D-A. Clevert, T. Unterthiner, S. Hochreiter, "Fast and accurate deep network learning by exponential linear units (ELUs)", ICLR 2016, 2016. 